\title{
Clinical applications of the Model for End-Stage Liver Disease (MELD) in hepatic medicine
}

\author{
This article was published in the following Dove Press journal: \\ Hepatic Medicine: Evidence and Research \\ 8 February 2013 \\ Number of times this article has been viewed
}

\section{Tsang Lau \\ Jawad Ahmad}

Division of Liver Diseases, Mount Sinai School of Medicine, New York, USA
Correspondence: Jawad Ahmad

Division of Liver Diseases,

Mount Sinai School of Medicine,

I Gustave L Levy Place, Box I 104

New York, NY 10029, USA

$\mathrm{Tel}+\mid \mathrm{I}$ | $224 \mid 8035$

$\mathrm{Fax}+\mathrm{I} 2127317340$

Email jawad.ahmad@mountsinai.org

\begin{abstract}
The Model for End-Stage Liver Disease (MELD) score incorporates serum bilirubin, creatinine, and the international normalized ratio (INR) into a formula that provides a continuous variable that is a very accurate predictor of 90-day mortality in patients with cirrhosis. It is currently utilized in the United States to prioritize deceased donor organ allocation for patients listed for liver transplantation. The MELD score is superior to other prognostic models in patients with end-stage liver disease, such as the Child-Turcotte-Pugh score, since it uses only objective criteria, and its implementation in 2002 led to a sharp reduction in the number of people waiting for liver transplant and reduced mortality on the waiting list without affecting posttransplant survival. Although mainly adopted for use in patients waiting for liver transplant, the MELD score has also proved to be an effective predictor of outcome in other situations, such as patients with cirrhosis going for surgery and patients with fulminant hepatic failure or alcoholic hepatitis. Several variations of the original MELD score, involving the addition of serum sodium or looking at the change in MELD over time, have been examined, and these may slightly improve its accuracy. The MELD score does have limitations in situations where the INR or creatinine may be elevated due to reasons other than liver disease, and its implementation for organ allocation purposes does not take into consideration several conditions that benefit from liver transplantation. The application of the MELD score in prioritizing patients for liver transplantation has been successful, but further studies and legislation are required to ensure a fair and equitable system.
\end{abstract}

Keywords: MELD score, liver transplantation

Cirrhosis is typically a progressive condition characterized by marked fibrosis and nodule formation in the liver due to a number of causes. It is usually irreversible and led to over 30,000 deaths in the United States in 2009, making it the 12th leading cause of mortality. ${ }^{1}$ Cirrhotic patients can have well-compensated disease with little or no symptoms or present with decompensated disease, including ascites, encephalopathy, or gastrointestinal bleeding, due to portal hypertension. These latter patients are candidates for liver transplantation (LT). ${ }^{2}$

There are currently 15,000 patients awaiting LT in the United States, and only 6000-6500 transplants are performed annually; meanwhile, there is a $10 \%$ rate of death on the waiting list. ${ }^{3}$ Historically, allocation of deceased donor (DD) organs for LT was based primarily upon the amount of time a patient spent on the waiting list and subjective measures of disease severity. In 1998, the US Department of Health and Human Services issued its "Final Rule," calling on the transplant community to establish a set of objective criteria in prioritizing patients for transplant that were submit your manuscript $\mid$ www.dovepress.com

Dovepress

http://dx.doi.org// 0.2147/HMER.S9049
Hepatic Medicine: Evidence and Research 2013:5 I-I0

(C) 2013 Lau and Ahmad, publisher and licensee Dove Medical Press Ltd. This is an Open Access article which permits unrestricted noncommercial use, provided the original work is properly cited. 
most at need. ${ }^{4}$ Subsequently, the Model for End-Stage Liver Disease (MELD) was developed and adapted as a prognostic tool in advanced liver disease and is now used by UNOS to prioritize DD organ allocation for patients listed for $\mathrm{LT}^{5}$ The validity of the MELD score has since been shown in a variety of clinical scenarios to prognosticate the outcome in patients with advanced liver disease.

\section{Natural history and clinical presentation of end-stage liver disease}

Cirrhosis is the end-stage of a variety of chronic liver diseases, including viral or autoimmune hepatitis, metabolic and alcoholic liver disease, and fatty liver disease. The course of chronic liver disease is variable and not all patients will develop cirrhosis. However, if present there is a wide range of clinical and laboratory presentations, which can vary depending on the etiology. The natural history starts with a stable "compensated" phase. Many patients with compensated cirrhosis are asymptomatic and can remain in this stage with only slow progression for many years. In a cohort of patients with well-compensated cirrhosis from chronic hepatitis C (HCV) infection, life expectancy was found to be relatively high with a 5-year survival of $91 \% .{ }^{6}$ Patients with nonalcoholic steatohepatitis (NASH)-related cirrhosis and cryptogenic cirrhosis (CC) appear to have slower disease progression than patients with HCV-associated cirrhosis while waiting for LT. ${ }^{7}$ Alcoholic cirrhosis has a similar natural history to HCV-related cirrhosis, with abstinence from alcohol being extremely important in improving survival of these patients. ${ }^{8}$

Another potential risk in patients with cirrhosis is the development of hepatocellular carcinoma (HCC), which varies according to etiology of the liver disease; viral hepatitis and some metabolic diseases confer a higher risk than NASH or CC. The development of HCC can herald the onset of decompensation but typically is asymptomatic and often identified on surveillance abdominal imaging.

Decompensated cirrhosis is characterized by manifestation of the complications of portal hypertension and/or liver dysfunction. The main clinical presentations include fatigue, abdominal distension from ascites, gastrointestinal bleeding from esophageal or gastric varices, and confusion due to portosystemic encephalopathy. The development of other complications can lead to a rapid decline, particularly spontaneous bacterial peritonitis and hepatorenal syndrome. Patients can present with cardiorespiratory complaints, such as dyspnea, which may be a manifestation of shunting in the pulmonary vasculature due to hepatopulmonary syndrome or portopulmonary hypertension. Once clinical decompensation has occurred, the prognosis is poor, with 50\% 5-year survival. ${ }^{6}$ Mortality is almost doubled when compared to patients with compensated cirrhosis.

In managing patients with decompensated cirrhosis, drug therapy for ascites, PSE and prophylaxis of variceal hemorrhage are the mainstay, but LT provides the only curative treatment option with excellent long-term results. However, the paucity of DD organs highlights the importance of an equitable organ allocation process. Up until 2002 a major determinant of a patient's priority on the LT waiting list was accumulated waiting time and hospitalization status. Patients in the intensive care unit (ICU) would receive priority, followed by non-ICU hospitalized patients and then outpatients. Assessment of the severity of illness also played a role and utilized the Child-Turcotte-Pugh (CTP) score.

\section{CTP score}

The CTP score was originally developed for stratifying the severity of liver disease. In 1964, Child and Turcotte published a classification system as a tool to determine the preoperative risk of portosystemic shunt surgery for patients with variceal bleeding. It took into account five factors, including ascites, encephalopathy, nutritional status, and serum levels of bilirubin and albumin. ${ }^{9}$ In 1973, Pugh et al modified this system and replaced nutritional status with prothrombin time (PT) and assigned a score ranging from 1 to 3 for each variable. ${ }^{10}$ This modified score was eventually expanded to predict outcomes in cirrhotic patients undergoing surgery. In 1998, UNOS also modified its listing criteria to include the CTP score to stratify potential liver recipients.

The CTP score has several limitations, however, especially with respect to potential use for prioritizing possible LT recipients. One major limiting factor is that it uses two subjective parameters in its calculation, portosystemic encephalopathy and severity of ascites. The interpretation of these symptoms is reliant on physical examination and is subject to differing interpretations among individual clinicians. In addition, both level of encephalopathy and ascites are subject to iatrogenic manipulation with medical therapies, such as lactulose, albumin, and diuretics. A second limiting factor of CTP is that renal function, a valuable prognostic marker in cirrhosis, is not taken into account. ${ }^{11,12}$

\section{Aim and purpose of MELD}

In the late 1990 s it became apparent that there were major inequalities in the distribution of DD organs for LT, with time on the waiting list and geographic location playing an 
important role. In addition, the various categories patients were placed in while waiting were based on relatively subjective CTP scores and hospitalization status. This led to discrepancies in waiting time across geographic regions and suggested that patients' chances of receiving a transplant were affected by where they lived. ${ }^{13}$ In 1998, the United States Department of Health and Human Services issued the "Final Rule" regulation, stating that the allocation of scarce DD livers should be based primarily on medical urgency and less emphasis should be placed on keeping organs in the local procurement area. ${ }^{14}$ The Institute of Medicine reviewed the impact of the final rule and recommended that the size of organ allocation areas be increased to a population base of 9 million people and disease severity should be based on an objective point system and waiting time should not be a factor. ${ }^{4}$

The precursor of the MELD score was introduced in an article published in 2000 by Malinchoc et al as a model to predict survival in patients with complications of portal hypertension undergoing elective placement of transjugular intrahepatic portosystemic shunts. ${ }^{15}$ Based upon a multivariate analysis investigating many predetermined variables, the authors identified four variables that had an independent and significant impact on short-term, 3-month survival. These variables included serum bilirubin, creatinine, the international normalized ratio (INR), and the cause of cirrhosis (alcoholic and cholestatic versus other causes). In 2001, the same group slightly modified this risk score into the MELD score, which incorporated serum bilirubin, creatinine, and INR, without the etiology of cirrhosis. ${ }^{16}$ The MELD score formula is: $3.8\left[\log _{\mathrm{e}}\right.$ serum bilirubin $\left.(\mathrm{mg} / \mathrm{dL})\right]+11.2\left[\log _{\mathrm{e}}\right.$ $\mathrm{INR}]+9.6\left[\log _{\mathrm{e}}\right.$ serum creatinine $\left.(\mathrm{mg} / \mathrm{dL})\right]+6.4$. The accuracy of the MELD score was evaluated with the "concordance statistic," or $c$-statistic, in which a $c$-statistic of $>0.7$ indicates a test with reasonable clinical utility, while a $c$-statistic $>0.8$ implies a strong diagnostic test. The $c$-statistic for MELD in this study for 3-month survival was almost always $>0.8$ in all cirrhotic patients studied. The addition of other complications of cirrhosis, such as ascites, encephalopathy, variceal bleeding, and spontaneous bacterial peritonitis, did not improve its diagnostic accuracy. The authors concluded that the MELD score was superior to the CTP score and proposed that it be used in the prioritization for the allocation of DD organs for LT.

On February 27, 2002, the MELD score was adopted by UNOS in prioritizing allocation of DD organs for LT in the United States. The major advantage of the MELD score over the CTP score was its ability to provide a continuous score based upon objective parameters in order to provide donor organs to the sickest patients first. In a 2003 study, Wiesner et al confirmed the predictive accuracy of the MELD score in predicting the 3-month mortality among 311 patients with chronic liver disease on the pretransplant waiting list. ${ }^{17}$ The results showed that the mortality of patients with end-stage liver disease while on the waiting list was directly proportional to the MELD score at the time of listing (Figure 1). The authors concluded that the MELD score was superior to the CTP score in predicting 3-month mortality with a $c$-statistic of 0.83 for MELD and 0.76 for CTP.

For many years the number of people waiting for LT had been steadily rising, but the implementation of the MELD score resulted in an immediate $12 \%$ decrease in the total number of new candidates listed for transplantation (Figure 2). ${ }^{18}$ This likely was partly because waiting time was no longer a factor in allocation, removing the incentive to list patients with low MELD scores in order to gain waiting time. Similarly, by transplanting sicker patients, the steady rise in deaths on the waiting list was reversed by $3.5 \%$ in the first year of MELD (Figure 3), with an overall reduction in median waiting time and no adverse effect on early posttransplant survival. ${ }^{19}$

UNOS has established eleven geographic regions for administrative purposes. Within these regions there are a total of 58 organ procurement organizations that are tasked with DD organ recovery in their local area. Organs are allocated initially locally, then regionally, and finally nationally depending on the MELD score of patients on the waiting list (which is subdivided by blood type).

An important study by Merion et al ${ }^{20}$ showed that patients with MELD $<15$ had a greater risk of mortality at 1 -year posttransplant as compared to similar patients with MELD $<15$ who remained on the waiting list. The

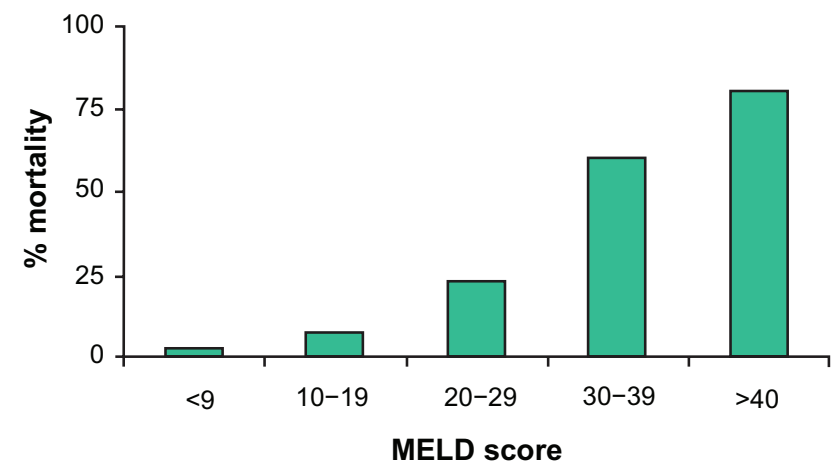

Figure I Three-month mortality based on listing MELD in patients on the OPTN waiting list.

Reprinted from SRTR/OPTN annual report 2003. Available at http://www.srtr.org. Abbreviations: MELD, Model for End-Stage Liver Disease; SRTR, Scientific Registry of Transplant Recipients; OPTN, Organ Procurement and Transplantation Network. 


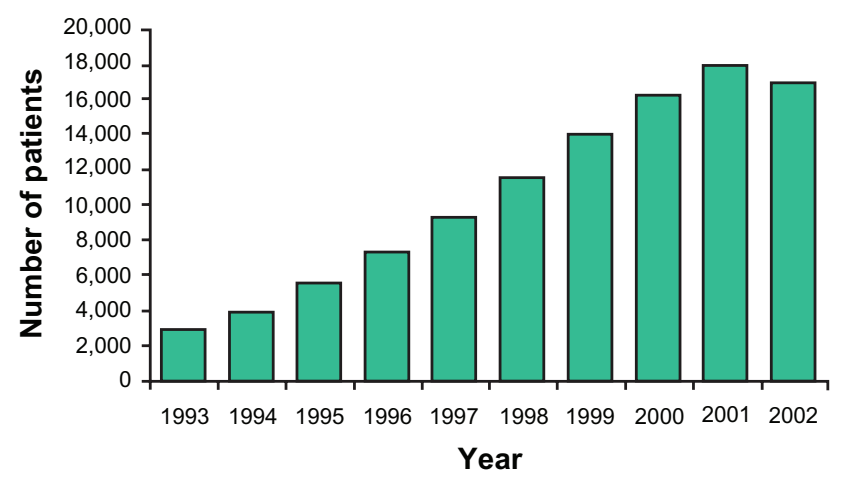

Figure 2 Number of patients on the liver waiting list, 1993-2002. Reprinted from SRTR/OPTN annual report 2003. Available at http://www.srtr.org. Abbreviations: SRTR, Scientific Registry of Transplant Recipients; OPTN, Organ Procurement and Transplantation Network.

Organ Procurement and Transplantation Network (OPTN) implemented the "Share-15" policy in January 2005 with the intent to provide sicker patients (MELD $>15)$ within a larger region priority over local patients with MELD $<15$. The implementation of this policy resulted in a $36 \%$ decrease in the proportion of LT recipients with MELD score $<15$ and reduced variability in the MELD score at the time of transplant across the country. ${ }^{21}$ Under the current system, ${ }^{22}$ the distribution sequence for a donor liver would be as follows: (1) local status 1, (2) OPTN region status 1, (3) local MELD $\geq 15$, (4) OPTN region MELD $\geq 15$, (5) local MELD $<15$, (6) OPTN region $<15$, (7) national status 1 , (8) national any MELD.

Using data from the Scientific Registry of Transplant Recipients (SRTR) on 28,165 adult liver transplant candidates wait listed between 2001 and 2005, Schaubel et al ${ }^{23}$ estimated the survival benefit from LT taking into account the candidate MELD score and deceased donor risk index (DRI). The DRI is a marker of DD organ quality incorporating several fac-

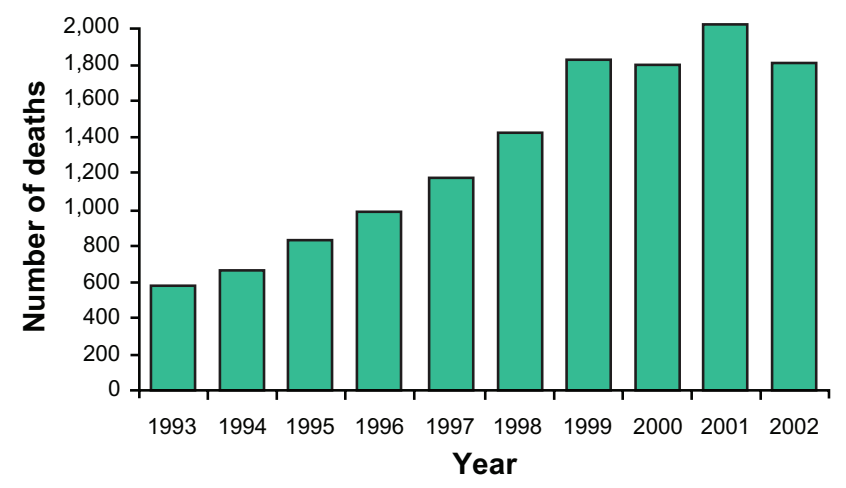

Figure 3 Number of deaths on the liver waiting list, 1993-2002.

Reprinted from SRTR/OPTN annual report 2003. Available at http://www.srtr.org. Abbreviations: SRTR, Scientific Registry of Transplant Recipients; OPTN, Organ Procurement and Transplantation Network. tors with higher values reflecting a "poorer" quality organ with a greater risk of graft failure. Compared to high-MELD recipients, low-MELD recipients (MELD 6-8) who received high-DRI organs experienced significantly higher mortality (hazard ratio $=3.70 ; P<0.0005$ ). On the other hand, all recipients with MELD $\geq 20$ had a significant survival benefit from transplantation, regardless of the DRI. The authors concluded that transplantation of high-DRI organs is effective for high- but not low-MELD candidates and urged for careful pairing of organs and recipients, particularly in those with low MELD scores. This led credence to the idea that transplanting low MELD score patients may impose higher risk than benefit, which favors the use of a broader organ sharing system.

Although the Share-15 policy may help to reduce regional variability in MELD at transplant, the 2010 SRTR/OPTN annual report ${ }^{24}$ showed that there remains a marked disparity in median MELD scores for adult DDLT among the eleven regions (Figure 4). This translates into vast differences in waiting time (Figure 5) among patients from various regions and even between different centers. ${ }^{25}$ Given these circumstances, if insurance and financial resources permit, some patients opt to move to a region with a lower median wait time. These inequalities show that the allocation system needs to continuously be reexamined and that there may still be improvements that can be made.

\section{Other applications of MELD Surgical risk}

Patients with cirrhosis are at increased risk for perioperative morbidity and mortality with all types of surgery. The CTP score was created and has been traditionally applied to stratify surgical risks in patients with chronic liver disease. However, recent studies have shown that the MELD score is equally good, if not better, at predicting operative mortality for patients with cirrhosis undergoing cardiac and noncardiac surgery. A retrospective analysis by Teh et al showed that in 772 patients with cirrhosis who underwent major gastrointestinal surgery $(n=586)$, orthopedic surgery $(n=107)$, or cardiovascular surgery $(\mathrm{n}=79)$, MELD was a strong predictor of mortality at 30 days, 90 days, and persisting throughout a 20-year follow-up period. ${ }^{26}$ Postsurgical mortality at 30 days was $5.7 \%$ for MELD score $\leq 8$, while it was $>50 \%$ for MELD score $\geq 20$. Multiple retrospective studies have come to similar conclusions showing higher postoperative mortality in various types of nontransplant surgery with rising MELD scores. ${ }^{27-29}$ 


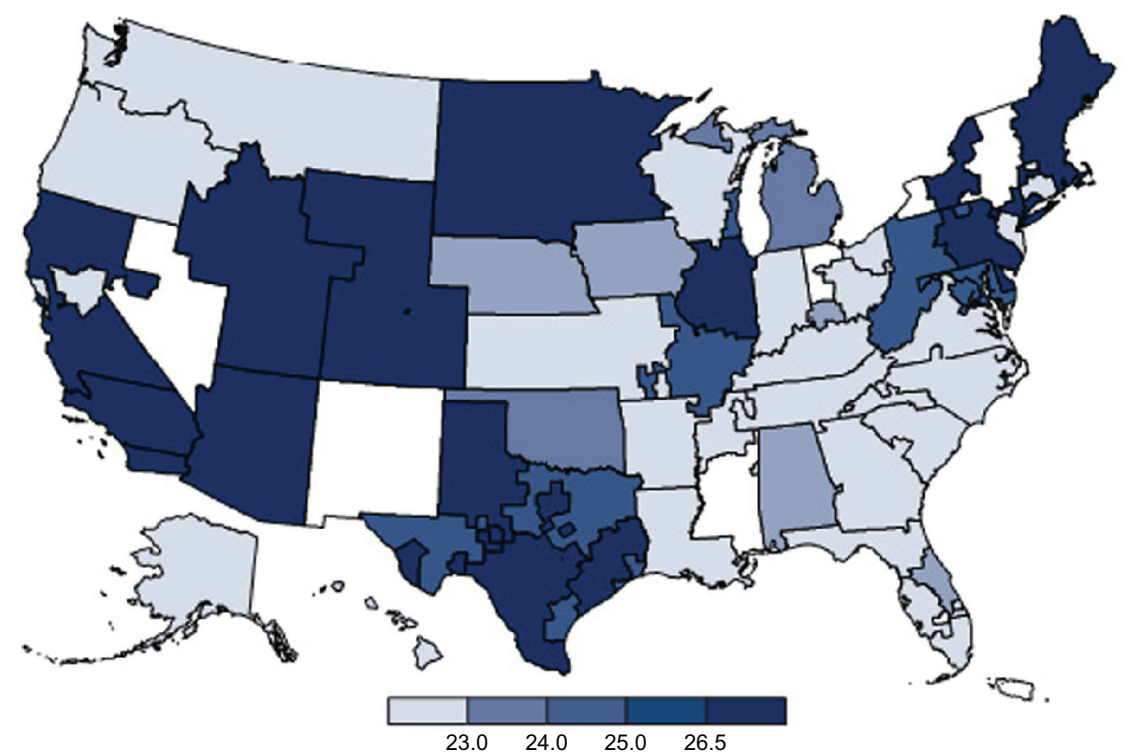

Figure 4 Median MELD score at time of transplant for adult deceased donor liver transplants, by donor service area in the United States, 2009. Reprinted from SRTR/OPTN annual report 2010. Available at http://www.srtr.org.

Abbreviations: MELD, Model for End-Stage Liver Disease; SRTR, Scientific Registry of Transplant Recipients; OPTN, Organ Procurement and Transplantation Network.

\section{Esophageal variceal bleeding}

Esophageal variceal bleeding is a life-threatening complication of cirrhosis, with studies showing that in-hospital mortality can be as high as $20 \%-25 \% .{ }^{30}$ In a retrospective study by Chalasani et al, MELD was used to evaluate mortality in 239 patients presenting with acute variceal bleeding.
MELD was predictive of in-hospital mortality with a $c$-statistic of $0.82,{ }^{31}$ a finding confirmed in several other studies. ${ }^{32-34}$

\section{Hepatorenal syndrome}

Hepatorenal syndrome (HRS) is defined by renal dysfunction in the absence of kidney disease in individuals with

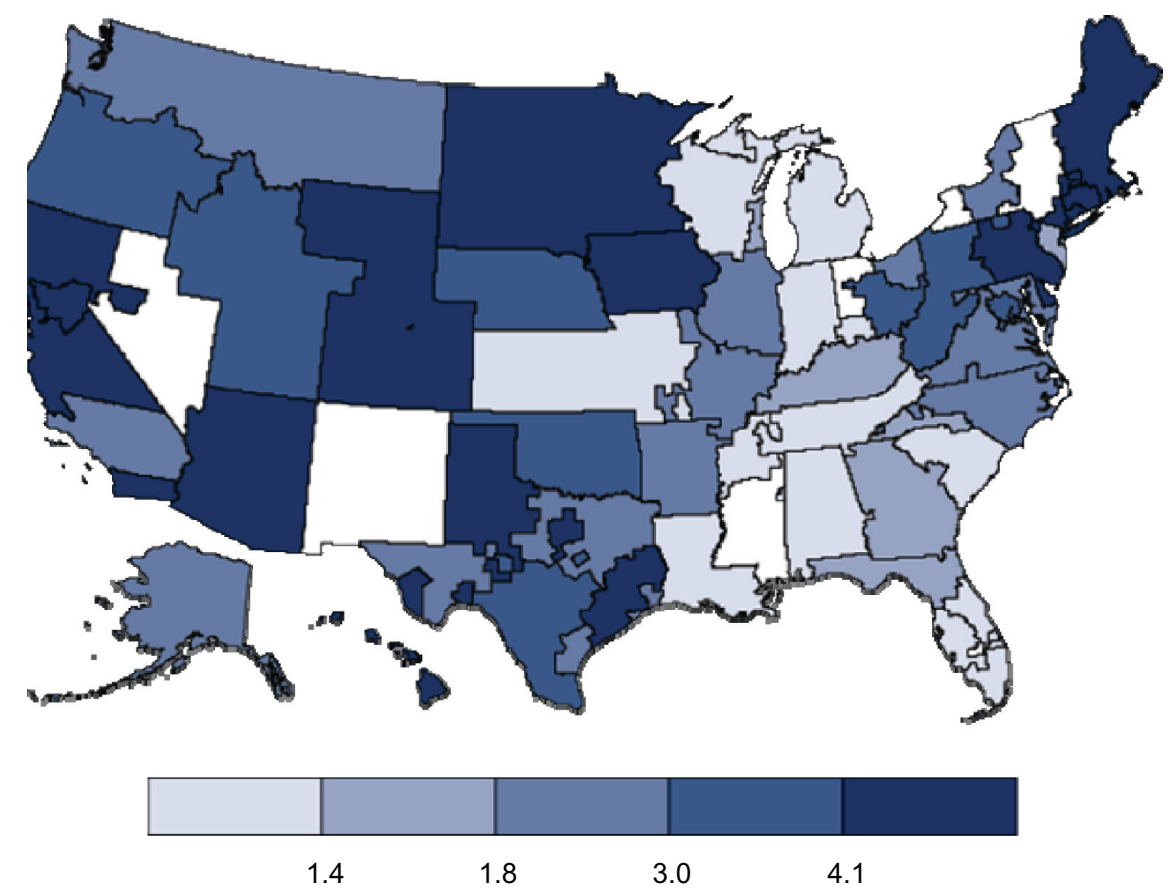

Figure 5 Median months to liver transplant for adult patients transplanted in 2009, by donor service area in the United States, 2009. Reprinted from SRTR/OPTN annual report 2010. Available at http://www.srtr.org.

Abbreviations: SRTR, Scientific Registry of Transplant Recipients; OPTN, Organ Procurement and Transplantation Network. 
end-stage liver disease, alcoholic hepatitis, or fulminant hepatic failure from any cause. HRS is subdivided into two types $^{35}$ depending on the acuity of the renal dysfunction. HRS type 1 presents with a doubling of the serum creatinine concentration (above $2.5 \mathrm{mg} / \mathrm{dL}$ ) and reduction of creatinine clearance $(\mathrm{CrCl})$ by $50 \%$ (or $<20 \mathrm{~mL} / \mathrm{min}$ ) in less than 2 weeks. It is associated with very poor outcomes with 1 -month mortality exceeding $50 \% .{ }^{35}$ HRS type 2 is defined by an increase in serum creatinine level to $>1.5 \mathrm{mg} / \mathrm{dL}$ (or $\mathrm{CrCl}<40 \mathrm{~mL} / \mathrm{min}$ ) and a urine sodium level $<10 \mathrm{mmol} / \mathrm{L}$. HRS type 2 has a less progressive course but still has a 6-month mortality of $50 \% .^{35,36}$

The only curative measure for both types is LT. In a study of 105 patients with cirrhosis and HRS, Alessandria et al showed that HRS type and MELD score were independent predictors of outcome. ${ }^{37}$ All patients with type 1 HRS had a high MELD score $(>20)$ and showed an extremely poor outcome with 1-month median survival. By contrast, survival in patients with type 2 HRS was longer and independently related to MELD score. Those with MELD score $\geq 20$ had a median survival of 3 months versus 11 months in those with MELD $<20(P<0.002)$. This is not surprising since creatinine is heavily weighted in the MELD score equation but underscores the fact that renal dysfunction in patients with liver disease is an ominous sign.

\section{Acute liver failure/UNOS status I}

Currently patients with acute liver failure are assigned a UNOS status 1 priority for LT, which means they are listed ahead of all patients listed with a MELD score on the waiting list. ${ }^{32}$ Patients listed as status 1 must fulfill one of the following criteria: (1) fulminant hepatic failure (FHF), defined as the onset of hepatic encephalopathy within 8 weeks of the first symptoms of liver failure with no pre-existing liver disease; (2) nonfunction of a transplanted liver within 7 days of implantation; (3) hepatic artery thrombosis in a transplanted liver within 7 days; (4) acute decompensated Wilson disease. Patients must fulfill one of the above criteria in addition to being hospitalized in the ICU with a life expectancy to be less than 7 days to be qualified for the emergent listing. Their ranking within this category depends upon blood type, geographic location of the patient, and waiting time on the transplantation list.

Yantorno et al demonstrated that in a series of 120 patients with FHF, the $c$-statistic for the MELD score in predicting poor prognosis in adults with FHF was $>0.95$ and was superior to preexisting prognostic tools, such as the King's College Criteria. ${ }^{38}$ When examining acetaminophen-induced hepatic failure,
Schmidt and Larsen showed that a higher MELD score on the first day after overdose was strongly associated with development of hepatic encephalopathy and subsequent FHF. ${ }^{39}$

In a retrospective analysis of 720 UNOS status 1 patients from 1999-2002, Kremers et $\mathrm{al}^{40}$ noted that an increased MELD score had a negative correlation with survival in patients with FHF not associated with acetaminophen toxicity (FHF-NA). The same group of patients also experienced the greatest survival benefit associated with LT. However, similar correlations were not observed in acute liver failure due to other status 1 indications, such as primary graft nonfunction or hepatic artery thrombosis. These findings suggest that the MELD score may be useful for prioritizing organ allocation for LT in some cases of FHF, but the failure of subsequent studies to confirm these findings $\mathrm{s}^{41,42}$ means that the current system of a status 1 assignment is preferred.

\section{Alcoholic hepatitis}

Alcoholic hepatitis $(\mathrm{AH})$ is characterized by acute or acute on chronic hepatocyte inflammatory injury from chronic excessive alcohol consumption. Clinical signs can vary from asymptomatic with mild elevation of liver chemistries to development of acute liver failure. According to the National Institute on Alcohol Abuse and Alcoholism, mortality in severe cases can be as high as $50 \% .{ }^{43}$ The discriminant function (DF) has traditionally been used to predict shortterm survival in patients with $\mathrm{AH}$ and is calculated by the following equation: $\mathrm{DF}=4.6[\mathrm{PT}$ in seconds - control $\mathrm{PT}]+$ serum bilirubin $(\mathrm{mg} / \mathrm{dL})$. A score $\geq 32$ has been used to predict significant mortality and to determine the need for glucocorticoids or pentoxifylline therapy. ${ }^{44}$ However, the DF is limited in its applicability as it relies largely upon measurement of the control PT, which is subject to variability among different medical laboratories.

A retrospective study by Dunn et al examined a cohort of 73 patients diagnosed with $\mathrm{AH}$. The $c$-statistic comparing the prognostic validity of MELD and DF in AH was comparable for 30-day as well as 90-day mortality. ${ }^{45}$ A MELD score of $\geq 21$ had $75 \%$ sensitivity and $75 \%$ specificity for predicting 90-day mortality. The investigators concluded that MELD is a useful clinical tool with which to gauge mortality and guide treatment decisions, although they did not recommend an exact MELD score cut-off point to initiate therapy and felt more studies were needed. A similar study of 202 patients admitted for AH found the MELD score to be superior to the DF score in predicting in-hospital mortality. MELD scores were recorded at two time points, including admission and at the first week, along with the interval 
change in score. All three of these factors were found to be independently associated with in-hospital mortality. The first week MELD score cut-off at 20 had the highest sensitivity and specificity in predicting mortality. ${ }^{46}$ In patients with $\mathrm{AH}, \mathrm{MELD} \geq 20$ appears to be an independent predictor of development of septic events, HRS and short-term, inhospital, liver-related mortality. Hence, this group of patients should be actively screened for sepsis and monitored closely for development of HRS. ${ }^{47}$

\section{Problems and limitation of MELDs}

The MELD score has proven to be a useful tool to assess the severity of liver disease. However, since it is based only on three objective laboratory variables, it can be affected by other clinical situations that can alter these variables. For example, an elevated INR can be secondary to warfarin use. In addition, patients may have an elevated serum creatinine from underlying kidney disease rather than hepatorenal syndrome. Serum bilirubin can be elevated in cases of sepsis or hemolysis. Any of these issues can increase the MELD score and overestimate the severity of liver disease.

Conversely, there are situations where the calculated MELD score does not take into account other conditions related to the underlying liver disease that are associated with an overall poor prognosis without LT. For organ allocation purposes in LT, this has led to the concept of the "MELD exception." The most common example is HCC, where almost all patients have pre-existing chronic liver disease and cirrhosis. Many patients with HCC do not demonstrate the degree of hepatic synthetic dysfunction necessary to give them a high enough MELD score to get priority for transplant. A seminal Italian study showed that LT in selected patients with early HCC leads to comparable long-term survival to those without malignancy. ${ }^{48}$ Early HCC determined by these Milan criteria, defined as either a single lesion $\leq 5 \mathrm{~cm}$ or up to three separate lesions but none $>3 \mathrm{~cm}$, without evidence of gross vascular invasion, and no regional nodal or distant metastases, is now a common reason for granting a MELD exception. Multiple studies have validated the use of the Milan criteria for improving survival in patients with HCC. ${ }^{49-51}$ Under current UNOS policy, patients who fit the Milan criteria are assigned an initial MELD exception score of 22 points, with an interval increase every 3 months on the waiting list to reflect a corresponding increase in the estimated 3-month mortality rate.

Other situations where MELD exceptions are given include recurrent episodes of cholangitis in patients with primary sclerosing cholangitis, hepatopulmonary syndrome (characterized by $\mathrm{PaO}_{2}<60 \mathrm{mmHg}$ on room air), and portopulmonary hypertension (characterized by a mean pulmonary artery pressure $\geq 35 \mathrm{mmHg}$ at diagnosis that must be maintained at $<35 \mathrm{mmHg}$ with treatment). Other rare conditions where there may not be any underlying liver dysfunction but where LT is the best treatment option can also benefit from a MELD exception, such as polycystic liver disease, familial amyloidosis, and primary oxaluria. ${ }^{22}$ Patients listed with the MELD exception typically receive a $10 \%$ increase in their MELD score every 3 months while on the waiting list (Table 1). Each individual transplant center may also submit petitions to a review board in their UNOS region for additional MELD points for patients whose medical urgency is not reflected by their calculated MELD score.

\section{Newer variations of MELD}

Although the MELD score is a good predictor of mortality in patients with chronic liver disease, several studies have looked at adding other variables or monitoring the change in MELD over time to improve its accuracy.

\section{$\triangle M E L D$}

Measuring the change in MELD scores over time ( $\triangle$ MELD) is thought to estimate the rate of loss of residual liver function. In a study by Huo et al, 351 cirrhotic patients were prospectively followed and $\triangle$ MELD showed better prognostic value for mortality compared to initial MELD and CTP scores at 6- and 12-month follow-up. ${ }^{52}$ The magnitude and direction of change in MELD score during the previous 30 days also appears to be a significant independent mortality predictor. ${ }^{53}$

Table I Acceptable indications for a MELD exception in the United States

\begin{tabular}{ll}
\hline Condition & $\begin{array}{l}\text { Typical MELD } \\
\text { exception points }\end{array}$ \\
\hline Hepatocellular carcinoma (within Milan criteria) & 22 \\
Hepatopulmonary syndrome & 22 \\
Cholangiocarcinoma & $*$ \\
Cystic fibrosis & $*$ \\
Familial amyloid polyneuropathy & $*$ \\
Primary hyperoxaluria & 28 \\
Portopulmonary hypertension & $*$ \\
Repeated cholangitis in PSC & $*$ \\
Polycystic liver disease & $*$ \\
Metastatic neuroendocrine tumor & $*$ \\
\hline
\end{tabular}

Notes: All conditions have to meet specified criteria to qualify for an exception. *The initial MELD exception is determined on a regional basis by the regional review board. Other conditions can be appealed for based on regional guidelines.

From http://optn.transplant.hrsa.gov/PoliciesandBylaws2/policies/pdfs/policy_8.pdf. (http://www.optn.org).

Abbreviations: MELD, Model for End-Stage Liver Disease; PSC, primary sclerosing cholangitis. 
Conversely, a negative $\triangle \mathrm{MELD}$ is likely to predict a subsequent lower risk of mortality. Therefore, the $\triangle \mathrm{MELD}$ score may have a better prognostic value for mortality than the MELD score at a single time point.

\section{MELDNa}

Another variation of MELD incorporates the serum sodium to give the MELD sodium (MELDNa). Hyponatremia is commonly associated with ascites, hepatorenal syndrome and is a marker for increased mortality among candidates for LT. ${ }^{54-58}$ Multiple studies have also shown that serum sodium levels may have a more significant correlation with mortality among patients with low MELD scores as compared to those with very high MELD scores. ${ }^{58,59}$ Pre-operative hyponatremia has also been found to be an independent predictor of mortality following LT. $^{60}$ These findings have led to proposals to incorporate serum sodium into the current MELD-based allocation system of DD livers in the United States.

Given these observations, Kim et al developed a new scoring system called MELDNa based upon data retrospectively collected from patients on the LT waiting list in $2005 .{ }^{61}$ The formula is calculated by: MELDNa $=$ MELD $-\mathrm{Na}-[0.025 *$ MELD * $(140-\mathrm{Na})]+140$, for sodium concentrations between 125 and $140 \mathrm{mEq} / \mathrm{L}$. The group then applied this formula prospectively to patients on the transplant list in 2006 and observed that MELDNa may be superior to MELD in predicting mortality.

In addition to its prognostic value in mortality among patients on the transplant waiting list, MELDNa has been applied to patients with acute hepatitis and was proven to have a better prognostic accuracy than the MELD score. ${ }^{62}$ Similarly, MELDNa was superior to the MELD score in predicting postoperative 90-day mortality in cirrhotic patients who underwent surgery under anesthesia. ${ }^{63}$ This new score is likely to be adopted by UNOS for DD organ allocation for LT in the future.

Recently the calculation of the MELD score has been slightly adjusted using updated coefficients for each of the variables in the equation and newer upper and lower levels for creatinine with a modest improvement in the $c$-statistic (refit MELD). ${ }^{64}$

\section{Conclusion}

Since the development of the MELD scoring system in 2001, MELD has been validated and applied to a wide spectrum of clinical scenarios. The implementation of MELD to prioritize DD organs for LT in 2002 led to a sharp reduction in waiting list registrations and reduced mortality on the waiting list without affecting posttransplant outcomes. ${ }^{62}$ The MELD score helps clinicians to risk stratifying various interventions on a daily basis in patients with cirrhosis in addition to influencing treatment options. The MELD scoring system does have its limitations, and further modification may improve its prognostic accuracy in the future.

\section{Disclosure}

The authors have no conflicts of interest to disclose.

\section{References}

1. Centers for Disease Control and Prevention. 2009 mortality data in patients with chronic liver disease or cirrhosis. Available from http:// www.cdc.gov/nchs/fastats/liverdis.htm. Accessed Sep 2012.

2. D'Amico G, Garcia-Tsao G, Pagliaro L. Natural history and prognostic indicators of survival in cirrhosis: a systematic review of 118 studies. J Hepatol. 2006;44:217-231.

3. Available at http://srtr.transplant.hrsa.gov/annual_reports/2010/ flash/03_liver/index.html\#/2/.Accessed September 9, 2012.

4. Institute of Medicine Committee on Organ Procurement and Transplantation Policy. Organ Procurement and Transplantation: Assessing Current Policies and the Potential Impact of the DHHS Final Rule. Washington, DC: National Academy Press; 1999:1-38.

5. Kamath PS, Kim WR. The model for end-stage liver disease (MELD). Hepatology. 2007;45:797-805.

6. Fattovich G, Giustina G, Degos F, et al. Morbidity and mortality in compensated cirrhosis type C: a retrospective follow-up study of 384 patients. Gastroenterology. 1997;112:463-472

7. O’Leary JG, Landaverde C, Jennings L, Goldstein RM, Davis GL. Patients with NASH and cryptogenic cirrhosis are less likely than those with hepatitis $\mathrm{C}$ to receive liver transplants. Clin Gastroenterol Hepatol. 2011;9:700-704.

8. Toshikuni N, Izumi A, Nishino K, et al. Comparison of outcomes between patients with alcoholic cirrhosis and those with hepatitis $\mathrm{C}$ virus-related cirrhosis. J Gastroenterol Hepatol. 2009;24:1276-1283.

9. Child CG, Turcotte JG. Surgery and portal hypertension. Major Probl Clin Surg. 1964;1:1-85.

10. Pugh R, Murray-lyon I, Dawson J. Transection of the oesophagus for bleeding oesophageal varices. Br J Surg. 1973;60:646-649.

11. Cooper G, Bellamy P, Dawson N. A prognostic model for patients with end-stage liver disease. Gastroenterology. 1997;113:1278-1288.

12. Fernandez-Esparrach G, Sanchez-Fueyo A, Gines P, et al. A prognostic model for predicting survival in cirrhosis with ascites. J Hepatol. 2001; 34:46-52.

13. Ubel PA, Caplan AL. Geographic favoritism in liver transplantationunfortunate or unfair? N Engl J Med. 1998;339:1322-1325.

14. Organ Procurement and Transplantation Network. Health Resources and Services Administration, HHS. Final Rule. Federal Register. 1999;64:56650-56661.

15. Malinchoc M, Kamath PS, Gordon FD, Peine CJ, Rank J, ter Borg PC. A model to predict poor survival in patients undergoing tran-jugular intrahepatic portosystemic shunts. Hepatology. 2000;31: 864-871.

16. Kamath PS, Wiesner RH, Malinchoc M, et al. A model to predict survival in patients with end-stage liver disease. Hepatology. 2001;33: 464-470.

17. Wiesner R, Edwards E, Freeman R, et al; United Network for Organ Sharing Liver Disease Severity Score Committee. Model for end-stage liver disease (MELD) and allocation of donor livers. Gastroenterology. 2003;124:91-96.

18. Freeman RB, Wiesner RH, Edwards E, Harper E, Harper A, Merion R, et al. Results of the first year of the new liver allocation plan. Liver Transpl. 2004;10:7-15 
19. Wiesner R, Lake J, Freeman R, Gish RG. Model for end-stage liver disease (MELD) exception guidelines. Liver Transpl. 2006; 12(Suppl):S85-S87.

20. Merion RM, Schaubel DE, Dykstra DM, Freeman R, Port FK, Wolfe RA. The survival benefit of liver transplantation. Am JTransplant. 2005;5:307-313.

21. Pomfret EA, Fryer JP, Sima CS, Lake JR, Merion RM. Liver and intestine transplantation in the United States, 1996-2005. Am J Transplant. 2007; 7:1376-1389.

22. Organ Procurement and Transplantation Network Policy 3.6. September 1, 2012. Available at: http://www.OPTN.org. Avaiiable at: http://optn.transplant.hrsa.gov/PoliciesandBylaws2/policies/pdfs/ policy_8.pdf. Accessed September 9, 2012.

23. Schaubel DE, Sima CS, Goodrich NP, Feng S, Merion RM.The survival benefit of deceased donor liver transplantation as a function of candidate disease severity and donor quality. Am J Transplant. 2008;8:419-425.

24. Organ Procurement and Transplantation Network (OPTN) and Scientific Registry of Transplant Recipients (SRTR). OPTN/SRTR 2010 Annual Data Report. Rockville, MD: Department of Health and Human Services, Health Resources and Services Administration, Healthcare Systems Bureau, Division of Transplantation; 2011. Available at: http://srtr. transplant.hrsa.gov/annual_reports/2010/chapter_index.htm. Accessed September 9, 2012.

25. Ahmad J, Bryce CL, Cacciarelli T, Roberts MS. Differences in access to liver transplantation: disease severity, waiting time, and transplantation center volume. Ann Intern Med. 2007;146:707-713.

26. Teh SH, Nagorney DM, Stevens SR, et al. Risk factors for mortality after surgery in patients with cirrhosis. Gastroenterology. 2007;132 1261-1269.

27. Costa BP, Sousa FC, Serôdio M, Carvalho C. Value of MELD and MELD-based indices in surgical risk evaluation of cirrhotic patients: retrospective analysis of 190 cases. World J Surg. 2009;33:1711-1719.

28. Ailawadi G, Lapar DJ, Swenson BR, et al. Model for endstage liver disease predicts mortality for tricuspid valve surgery. Ann Thorac Surg. 2009;87:1460-1468.

29. Northup PG, Wanamaker RC, Lee VD, Adams RB, Berg CL. Model for end-stage liver disease (MELD) predicts nontransplant surgical mortality in patients with cirrhosis. Ann Surg. 2005;242: 244-251.

30. Cerqueira RM, Andrade L, Correia MR, Fernandes CD, Manso MC. Risk factors for in-hospital mortality in cirrhotic patients with oesophageal variceal bleeding. Eur J Gastroenterol Hepatol. 2012;24:551-557.

31. Chalasani N, Kahi C, Francois F, Pinto A, Marathe A, Bini EJ, et al. Model for end stage liver disease (MELD) for predicting mortality in patients with acute variceal bleeding. Hepatology. 2002;35: 1282-1284.

32. Amitrano L, Guardascione MA, Bennato R, Manguso F, Balzano A. MELD score and hepatocellular carcinoma identify patients at different risk of short-term mortality among cirrhotics bleeding from esophageal varices. J Hepatol. 2005;42:820-825.

33. Bambha K, Kim WR, Pedersen R, Bida JP, Kremers WK, Kamath PS. Predictors of early rebleeding and mortality after acute variceal bleeding in patients with cirrhosis. Gut. 2008;57:814-820.

34. Chen WT, Lin CY, Sheen IS, Huang CW, Lin NT, Lin CJ, et al. MELD score can predict early mortality in patients with rebleeding after band ligation for variceal bleeding. World J Gastroenterol. 2011;17: 2120-2125.

35. Arroyo V, Gines P, Gerbes AL, et al. Definition and diagnostic criteria of refractory ascites and hepatorenal syndrome in cirrhosis. International Ascites Club. Hepatology. 1996;23:164-176.

36. Al Sibae MR, Cappell MS. Accuracy of MELD scores in predicting mortality in decompensated cirrhosis from variceal bleeding, hepatorenal syndrome, alcoholic hepatitis, or acute liver failure as well as mortality after non-transplant surgery or TIPS. Dig Dis Sci. 2011;56:977-987.

37. Alessandria C, Ozdogan O, Guevara M, et al. MELD score and clinical type predict prognosis in hepatorenal syndrome: relevance to liver transplantation. Hepatology. 2005;41:1282.
38. Yantorno SET, Trentadue JJ, Ruf AE, et al. MELD is superior to King's College and Clichy's criteria to assess prognosis in fulminant hepatic failure. Liver Transpl. 2007;13:822-828.

39. Schmidt LE, Larsen FS. MELD score as a predictor of liver failure and death in patients with acetaminophen induced liver injury. Hepatology. 2007;45:789-796.

40. Kremers WK, van Liperen M, Kim WR, et al. MELD score as a predictor of pretransplant and posttransplant survival in OPTN/UNOS status 1 patients. Hepatology. 2004;39:764-769.

41. Dhiman RK, Jain S, Maheshwari U, Bhalla A, Sharma N, Ahluwalia J, et al. Early indicators of prognosis in fulminant hepatic failure: an assessment of the Model for End-Stage Liver Disease (MELD) and King's College Hospital criteria. Liver Transpl. 2007;13:814-821.

42. Katoonizadeh A, Decaestecker J, Wilmer A, Aerts R, Verslype C, Vansteenbergen W, et al. MELD score to predict outcome in adult patients with non-acetaminophen-induced acute liver failure. Liver Int. 2007;27:329-334

43. Mann RE, Smart RG, Govoni R. The Epidemiology of Alcoholic Liver Disease. Available at: http://pubs.niaaa.nih.gov/publications/arh2723/209-219.htm. Accessed September 29, 2004.

44. Maddrey WC, Boitnott JK, Bedine MS, Weber FL Jr, Mezey E, White RI Jr. Corticosteroid therapy of alcoholic hepatitis. Gastroenterology. 1978;75:193-199.

45. Dunn W, Jamil LH, Brown LS, et al. MELD accurately predicts mortality in patients with alcoholic hepatitis. Hepatology. 2005;41:353-358.

46. Srikureja W, Kyulo NL, Runyon BA, Hu KQ. MELD score is a better prognostic model than Child-Turcotte-Pugh score or discriminant function score in patients with alcoholic hepatitis. $J$ Hepatol. 2005;42:700-706

47. Verma S, Ajudia K, Mendler M, Redeker A. Prevalence of septic events, type 1 hepatorenal syndrome, and mortality in severe alcoholic hepatitis and utility of discriminant function and MELD score in predicting these adverse events. Dig Dis Sci. 2006;51:1637-1643.

48. Mazzaferro V, Regalia E, Doci R, et al. Liver transplantation for the treatment of small hepatocellular carcinomas in patients with cirrhosis. N Engl J Med. 1996;334:693-699.

49. Figueras J, Jaurrieta E, Valls C, et al. Survival after liver transplantation in cirrhotic patients with and without hepatocellular carcinoma: a comparative study. Hepatology. 1997;25:1485-1489.

50. Min AD, Saxena R, Thung SN, et al. Outcome of hepatitis $\mathrm{C}$ patients with and without hepatocellular carcinoma undergoing liver transplant. Am J Gastroenterol. 1998;93:2148-2153.

51. Yoo HY, Patt CH, Geschwind JF, Thuluvath PJ. The outcome of liver transplantation in patients with hepatocellular carcinoma in the United States between 1988 and 2001: 5-year survival has improved significantly with time. J Clin Oncol. 2003;21:4329-4335.

52. Huo TI, Wu JC, Lin HC, et al. Evaluation of the increase in model for end-stage liver disease (DeltaMELD) score over time as a prognostic predictor in patients with advanced cirrhosis: risk factor analysis and comparison with initial MELD and Child-Turcotte-Pugh score. J Hepatol. 2005;42:826-832.

53. Merion RM, Wolfe RA, Dykstra DM, Leichtman AB, Gillespie B, Held PJ. Longitudinal assessment of mortality risk among candidates for liver transplantation. Liver Transplant. 2003;9:12-18.

54. Arroyo V, Colmenero J. Ascites and hepatorenal syndrome in cirrhosis: pathophysiological basis of therapy and current management. J Hepatol. 2003;38(Suppl 1):S69-S89.

55. Gines A, Escorsell A, Gines P, et al. Incidence, predictive factors, and prognosis of the hepatorenal syndrome in cirrhosis with ascites. Gastroenterology. 1993;105:229-236.

56. Ruf AE, Kremers WK, Chavez LL, Descalzi VI, Podesta LG, Villamil FG. Addition of serum sodium into the MELD score predicts waiting list mortality better than MELD alone. Liver Transpl. 2005;11: 336-343.

57. Heuman DM, Abou-Assi SG, Habib A, et al. Persistent ascites and low serum sodium identify patients with cirrhosis and low MELD scores who are high risk for early death. Hepatology. 2004;40:802-810. 
58. Biggins SW, Ray Kim W, Terrault N, et al. Evidence-based incorporation of serum sodium concentration into MELD. Gastroenterology. 2006;130:1652-1660.

59. Biggins SW, Rodriguez HJ, Bacchetti P, Bass NM, Roberts JP, Terrault NA. Serum sodium predicts mortality in patients listed for liver transplantation. Hepatology. 2005;41:32-39.

60. Dawwas MF, Lewsey JD, Neuberger JM, et al. The impact of serum sodium concentration on mortality after liver transplantation: a cohort multicenter study. Liver Transpl. 2007;13:1115-1124.

61. Kim R, Biggins SW, Kremers WK, et al. Hyponatremia and mortality among patients on the liver transplant waiting list. New Engl J Med. 2008;359:1018-1026.
62. Hsu CY, Lin HC, Huang YH, et al. Comparison of the model for endstage liver disease (MELD), MELD-Na and MELDNa for outcome prediction in patients with acute decompensated hepatitis. Dig Liver Dis. 2010;42:137-142.

63. Cho HC, Jung HY, Sinn DH, et al. Mortality after surgery in patients with liver cirrhosis: comparison of Child-Turcotte-Pugh, MELD and MELDNa score. Eur J Gastroenterol Hepatol. 2011;23:51-59.

64. Leise MD, Ray Kim W, Kremers WK, Larson JJ, Benson JT, Therneau TM. A revised model for end-stage liver disease optimizes prediction of mortality among patients awaiting liver transplantation. Gastroenterology. 2011;140:1952-1960.

\section{Publish your work in this journal}

Hepatic Medicine: Evidence and Research is an international, peerreviewed, open access journal covering all aspects of adult and pediatric hepatology in the clinic and laboratory including the following topics: Pathology, pathophysiology of hepatic disease; Investigation and treatment of hepatic disease; Pharmacology of drugs used for the treatment of hepatic disease. Issues of patient safety and quality of care will also be considered. The manuscript management system is completely online and includes a very quick and fair peer-review system, which is all easy to use. Visit http://www.dovepress.com/ testimonials.php to read real quotes from published authors.

Submit your manuscript here: http://www.dovepress.com/hepatic-medicine-evidence-and-research-journal 